\title{
ALGUNAS REFLEXIONES SOBRE LAGUNAS Y ANTINOMIAS EN PRINCIPIA IURIS*
}

\author{
Eugenio Bulygin \\ Universidad de Buenos Aires
}

RESUMEN. El autor reflexiona en torno al tratamiento que L. FERRAJOLI da a las antinomias y lagunas en Principia juris. Colateralmente aborda a su vez críticamente los comentarios que respecto del mismo aspecto de la obra ferrajoliana han sido formulados por J. J. MoREso. Respecto de ésta, BULYGIN cuestiona, principalmente, i) la distinción entre sistemas de un solo nivel normativo o sistemas nomoestáticos (que dan lugar a lo que llama el Estado legislativo de Derecho) y sistemas nomodinámicos, o de varios niveles normativos (Estado constitucional); ii) la idea de que las lagunas y antinomias sólo aparecen en los sistemas de más de un nivel normativo, no así en los de un solo nivel; iii) el postulado por el cual de aquello que no está permitida la comisión, está permitida la omisión, y iv) el postulado de que todo comportamiento supone una modalidad que lo califica deónticamente.

Palabras clave: FerRAJolı, antinomias, lagunas, Principia iuris.

ABSTRACT. The author reflects on the subject of L. FERRAJOLI's treatment of antinomies and gaps in Principia juris. At the same time, he critically approaches the comments formulated by J. J. MORESO on the same aspect of FERRAJOLI's work. With respect to FERRAJOLI's work, BULYGIN mainly questions, i) the distinction between systems with a single normative level, or nomostatic systems (which give rise to what he calls Legislative State of law) and systems with various normative levels or nomodynamic systems (Constitutional State); ii) the idea that gaps and antinomies only appear in systems of more than one normative level, and not in those of a single level; iii) the postulate which permits the ommission of actions whose commission is forbidden; and iv) the postulate by which all behaviour presupposes a modality which qualifies it deontically.

Keywords: FerRAJOLI, antinomies, loopholes, Principia iuris.

\footnotetext{
* Fecha de recepción: 1 de diciembre de 2008. Fecha de aceptación: 18 de diciembre de 2008.
} 


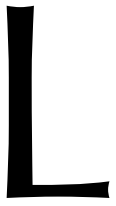

as líneas que siguen se refieren a un aspecto menor de la monumental obra de L. FERRAJOLI, que acaba de aparecer: Principia iuris (tres volúmenes, 2.735 pp., Roma-Bari: Editori Laterza, 2007). Muy poco tiempo después de su publicación, T. MAZZARESE organizó en Brescia un simposio sobre esta obra, del que no pude participar. Sin embargo, Tecla insistió que escribiera un ensayo para los actas del simposio y dada mi antigua amistad, tanto con la organizadora como con el autor, no pude negarme. Naturalmente, elegí el tema de lagunas y antinomias (sobre el cual algo he escrito) y comencé a escribir mis comentarios, cuando mi amigo J. J. MORESO me mandó su ensayo con la idea de estimularme, pero que tuvo un efecto contrario: me paralizó totalmente, pues trataba el mismo tema en que había pensado yo. Así lo comuniqué a Tecla, pero ella, lejos de abandonar su insistencia, la redobló con amenazas de horribles represalias, cosa que me obligó a reanudar mi trabajo. Una lectura más atenta me convenció de que no estaba totalmente de acuerdo ni con Ferrajoli, ni con Moreso. En lo que sigue, trato de articular mis dudas con la intención de entender mejor las ideas expuestas en el libro. Demás está decir que las eventuales críticas no implican en modo alguno una mella en mi enorme simpatía y admiración tanto por la persona de mi amigo Luigi como por su magnífica obra.

1. FERRAJOLI sostiene varias tesis que, me parece, requieren alguna aclaración y/o fundamentación mayor.

En primer lugar, distingue entre sistemas de un solo nivel normativo o sistemas nomoestáticos (que dan lugar a lo que llama el Estado legislativo de Derecho) y sistemas nomodinámicos o de varios niveles normativos (Estado constitucional). Esta distinción desempeña un papel muy importante en la obra de FERRAJOLI.

En segundo lugar, sostiene que las lagunas y las antinomias o contradicciones normativas sólo aparecen en los sistemas de más de un nivel normativo; no así en los en los sistemas de un solo nivel.

Tengo algunos reparos respecto de ambos puntos. Comparto la observación de MoRESO quien pone en duda de que el Estado legislativo de Derecho tenga un solo nivel normativo. Las leyes, los decretos reglamentarios, las resoluciones ministeriales o del Banco Central no parecen estar en el mismo nivel. Los problemas de adecuación que se plantean entre los decretos reglamentarios y las leyes no son esencialmente diferentes de los que se dan entre las leyes y las normas constitucionales.

Tampoco entiendo porqué FERRAJOLI llama nomoestáticos a los sistemas de un solo nivel normativo y reserva el nombre de nomodinámicos para los sistemas de varios niveles como el Estado constitucional. Por un sistema nomodinámico se entiende generalmente (siguiendo una idea de KELSEN) un sistema sujeto a cambios en el tiempo; en el sentido de que las normas que lo integran pueden ser cambiadas, a saber, nuevas normas pueden introducirse mediante actos de creación normativa, normas existentes pueden ser eliminadas mediante derogación o desuetudo y toda norma puede y suele ser tarde o temprano reformada, es decir, derogada parcialmente y completada con nuevas disposiciones. Esta última operación es una combinación de derogación y creación. En este sentido todo sistema jurídico es un sistema dinámico, pues para ser tal basta que haya autoridades creadoras y derogadoras de normas y esta condición es satisfecha por todos los sistemas jurídicos conocidos. Un sistema estático, en cambio, sería aquel que 
no está sujeto a cambios en el tiempo, como sucede, por ejemplo, con un sistema geométrico: una vez fijados sus axiomas o principios y las reglas de inferencia, el sistema no cambia, pues queda fijado para siempre. Parece evidente que FERRAJOLI usa los términos «nomoestático» y «nomodinamico» en un sentido especial, pero no se advierte la razón de este cambio.

2. La distinción tajante entre Estado legislativo y Estado constitucional lleva a FERRAJOLI a la tesis de que en los sistemas de un solo nivel normativo y, en particular, en el Estado legislativo no habría ni lagunas ni antinomias, lo cual parece implicar que tales sistemas son siempre completos y consistentes. Esto dio lugar a una severa crítica por parte de $\mathrm{MORESO}^{1}$. Creo, sin embargo, que corresponde hacer algunas observaciones aclaratorias.

En primer lugar, FERRAJOLI no niega que pueda haber lagunas y antinomias en un sistema de un solo nivel; lo que sucede es que él usa las nociones de laguna y antinomia en un sentido especial y es en este sentido en que sólo habría lagunas y antinomias en los sistemas de varios niveles. En efecto, FERRAJOLI define «antinomia» como «il vizio sostanziale dall' indebita decisione di una norma in contrasto con una norma sostanziale sulla produzione, la cui applicazione suppone l'anullamento della norma in contrasto» (D10.43, vol. I, p. 685), y «laguna» como «il vizio prodotto dall'indebita omissione della decisione di una norma richiesta da una norma sulla produzione, la cui applicazione suppone l'introduzione della norma mancante» (D10.44, vol. I, p. 686).

En este sentido no basta una mera contradicción entre dos normas para constituir una antinomia, ni una mera ausencia de una solución para un caso para dar lugar a una laguna. Son nociones mucho más restringidas, como el mismo FERRAJOLI aclara: «Le ridefinizioni che propongo sono detatte da una specifica finalitá esplicativa: la necesitá di identificare e di isolare quelle antinomie e quelle lacune che generano non qualunque problema di applicazione, ma solo problemi non solubili senza una modifica dell'ordinamento. Esse resringono perció il campo di denotazione dei due termini...» (vol. I, p. 685).

En consecuencia, FERRAJOLI distingue entre dos sentidos en que se puede hablar de lagunas y antinomias en el Derecho: el sentido fuerte (estricto o estructural, definido más arriba) y el sentido débil que corresponde a lo que comúnmente se entiende por «laguna» y «antinomia» (vol. I, p. 688), conceptos que han sido analizados, entre otros, en Normative Systems ${ }^{2}$. El problema de algunas y antinomias en sentido fuerte es el que examina KELSEN bajo el rótulo de «Conflictos entre normas de diferente grado» ${ }^{3}$. La solución de KELSEN para este problema es su famosa cláusula alternativa tácita, que difícilmente puede considerarse satisfactoria ${ }^{4}$.

Las características distintivas del sentido fuerte de esos defectos, son dos: primero, suponen la existencia de varios niveles normativos y segundo, no pueden ser elimina-

${ }^{1} \mathrm{~J}$. J. MORESO, «Ferrajoli o el constitucionalismo optimista» en este volumen.

2 C. E. AlchourRón y E. BulYgin, 1971: Normative Systems, Wien-New York: Springer Verlag, trad. Italiana de P. CHIASSONI y G. B. RATTI, 2005: Sistemi normativi. Introduzione alla metodologia della scienza giuridica, Torino: G. Giapichelli Editore.

${ }^{3}$ H. Kelsen, 1960: Reine Rechtslehre, 2. ${ }^{a}$ ed., Wien: Franz Deuticke, pp.271-282.

${ }^{4}$ Para la crítica de esa idea de KelSEN, $c f r$. E. BulYGIN, 1995: «Cognition and Interpretation of Law», en L. Gianformaggio y S. Paulson (eds.), Cognition and Interpretation of Law, Torino: G. Giapichelli Editore, pp. 11-35, y la discusión pp. 219-273 y 309-313 
dos o solucionados sin intervención de una autoridad jurídica porque requieren una modificación del orden jurídico, mientras que las lagunas y antinomias en sentido débil pueden ser eliminadas por el intérprete sin necesidad de la intervención de una autoridad.

El argumento que da FERRAJOLI para sostener esto último fue criticado por MORESO. Sucede que FERRAJOLI parece creer que los conocidos principios lex posterior y lex specialis son suficientes para eliminar las antinomias en un sistema de un solo nivel y que las lagunas en tales sistemas pueden ser colmadas mediante la aplicación analógica de las normas. A esto replica MORESO que los mencionados principios no siempre alcanzan para solucionar un caso de antinomia y que el argumento de la aplicación analógica para sostener la inexistencia de lagunas se parece al conocido argumento de CARRIÓ ${ }^{5}$, quien compara esta tesis con la afirmación de que un traje no tiene agujeros, porque un sastre puede remendarlos. Me parece, sin embargo, que el argumento central de FERRAJOLI no se apoya en la mera posibilidad de eliminar las antinomias y las lagunas mediante los principios lex posterior y lex specialis y la aplicación analógica de otras normas, sino en que esta eliminación puede ser practicada por el intérprete y no requiere la intervención de una autoridad normativa. Ésta es justamente una de las características distintivas de las antinomias y lagunas en el sentido fuerte o estructural. MORESO parece haber pasado por alto que FERRAJOLI, cuando habla de la eliminación de antinomias y lagunas en un sistema, se refiere al sentido débil en que cabe hablar de estos defectos y no al sentido fuerte, definido por él en las pp. 685-686 del vol. I.

Quedan, sin embargo, abiertas dos cuestiones: la primera consiste en saber si las antinomias y las lagunas en el sentido fuerte no pueden aparecer en un Estado legislativo de Derecho. Un posible ejemplo de laguna en sentido fuerte sería la falta de la reglamentación de una ley que expresamente exige que el poder ejecutivo la reglamente. Esto sería el caso de una laguna que sólo puede ser eliminada mediante un acto jurídico creador del Derecho y, por consiguiente, sería una laguna en el sentido fuerte o estructural. Pero aceptar esto implicaría rechazar la tesis de que tal sistema tiene un solo nivel normativo.

La segunda cuestión problemática es si la eliminación de normas en el caso de antinomias y la introducción de nuevas normas mediante razonamiento analógico no implica una modificación del orden jurídico. ALCHOURRÓN y MAKINSON ${ }^{6}$ han argumentado en forma convincente que en los casos en que las conocidas reglas lex superior, lex posterior y lex specialis no resuelven el problema de la antinomia, los jueces suelen imprimir un nuevo orden jerárquico al conjunto de normas en cuestión y luego aplican la norma que resulte superior a las demás. Este procedimiento equivale a la derogación de las normas descartadas por resultar jerárquicamente inferiores, lo que implica un cambio en el sistema jurídico, que no es un mero conjunto de normas, sino un conjunto jerárquicamente ordenado. Por consiguiente, el procedimiento de imprimir un nuevo orden al conjunto de normas es una tarea que rebasa los límites clásicos de la actividad judicial e invade la esfera de la competencia legislativa. Y algo muy parecido sucede

\footnotetext{
${ }^{5}$ G. R. CARRIÓ, 1965: Notas sobre derecho y lenguaje, Buenos Aires: Abeledo-Perrot.

${ }^{6}$ C. E. AlchourRón y D. MAKINSON, 1981: «Hierarchies of Regulations and Their Logic», en R. HiLPINEN (ed.), New Studies in Deontic Logic, Dordrecht: Reidel, pp. 125-148.
} 
cuando los jueces colman una laguna mediante la aplicación analógica; la norma aplicada es una nueva norma, aunque sea parecida a alguna norma existente, y esto también implica modificar el orden jurídico ${ }^{7}$.

3. El punto más discutible en la teoría de FERRAJOLI y que también critica MORESO son sus postulados P1 y P2 (vol. III, p. 22) que rezan así:

P1. De aquello que no está permitida la comisión, está permitida la omisión.

P2. Todo comportamiento supone una modalidad que lo califica deónticamente.

MORESO señala con razón que — dadas las definiciones de FERRAJOLI de las modalidades deónticas (permitido, prohibido, obligatorio y facultativo) - P1 ya implica P2, con lo cual P2 no sería un postulado independiente. En efecto, P1 dice que para toda conducta vale que o bien está permitida ella o bien está permitida su omisión, lo cual quiere decir que toda posible conducta está siempre afectada por una modalidad deóntica que es exactamente lo que dice P2. Dice Moreso: «De este modo, P1 ya dice que todo comportamiento está regulado por alguna modalidad deóntica, está prohibido o permitido. De acuerdo con esta tesis el derecho es completo porque es una verdad lógica que todos los comportamientos que no están prohibidos están permitidos (es decir, que todo lo que no está prohibido está permitido). Esta tesis parece avalada por la interdefinibilidad entre "No-prohibido" y "Permitido"». MORESO analiza las dos versiones del principio de que todo lo que no está prohibido, está permitido; en su versión débil (donde «permitido» significa «no prohibido») el principio es analíticamente verdadero, pero es compatible con la presencia de lagunas y en la versión fuerte, si «permitido» significa que hay una norma que permite, no es necesario. Concluye MoRESO que el principio de prohibición no es una justificación adecuada de P1 y que los dos postulados de FERRAJOLI (P1 y P2) no son verdades lógicas, sino formulaciones de ideales normativos.

Me parece que la crítica de MORESO en este punto es acertada. Indudablemente, si «permitido» quiere decir «no prohibido», el principio «lo que no está prohibido, está permitido» se reduce a una mera tautología: «lo que no está prohibido, no está prohibido». Cabe preguntarse, sin embargo, si el mero «no prohibido» es una modalidad deóntica. Si digo que todo lo que no es blanco es no-blanco, esto es verdad, pero ¿'es «noblanco» un color? Como ya observara ARISTÓTELES ${ }^{8}$, «no ser blanco» no significa lo mismo que «ser no-blanco».

Otra posibilidad sería interpretar el principio P1 como un requisito que deben cumplir los sistemas normativos para ser consistentes, porque un sistema que prohíbe tanto la realización de una conducta, como su omisión sería inconsistente. Pero no es una verdad lógica, porque los sistemas normativos pueden ser inconsistentes: nada impide que un sistema contenga contradicciones normativas o antinomias no resueltas, no sólo en el sentido fuerte de FERRAJOLI, sino también en el sentido ordinario, es decir, débil.

Por otra parte, los principios P1 y P2 parecen implicar que los sistemas jurídicos son completos, es decir, carentes de lagunas en el sentido débil, porque no puede dar-

${ }^{7}$ Cfr. E. Bulygin, 2005: «Creación y aplicación del derecho», en F. ATRIA et al., Lagunas en el derecho, Madrid-Barcelona: Marcial Pons, pp. 29-44, especialmente 43-44.

8 ARISTÓteles, Analytica Priora, L. 1, 46, 51b, 36. 
se el caso de una conducta no modalizada deónticamente. Pero las lagunas en sentido corriente son precisamente casos en los que una cierta conducta no está regulada por las normas, es decir, no está ni prohibida, ni permitida. Si esto es así, habría una contradicción: por un lado FERRAJOLI sostiene que las lagunas en sentido débil pueden ser colmadas por el intérprete mediante la aplicación analógica de otras normas. Pero el argumento de que el intérprete puede colmar las lagunas, lejos de probar su inexistencia, presupone que tales lagunas existen. Este argumento prueba que puede haber lagunas en sentido débil, pues si no las hubiera, no habría nada para colmar y no resultaría claro para qué sirve la aplicación analógica del derecho. Por otro lado, los principios P1 y P2 parecen precluir la posibilidad de tales lagunas, pues todo comportamiento está siempre modalizado deónticamente, como prohibido o como permitido. Coincido con MORESO en que estos dos principios no pueden ser considerados verdades lógicas y para ser tales deberían ser reformulados.

Como dije al comienzo, estas críticas son menores y no afectan la formidable construcción de Principia Juris, que es una de las obras más importantes que se han publicado en los últimos cincuenta años. 